www.jmscr.igmpublication.org Impact Factor 5.244

Index Copernicus Value: 83.27 ISSN (e)-2347-176x ISSN (p) 2455-0450 crossref DOI: https://dx.doi.org/10.18535/jmscr/v4i12.124

\title{
COPD: Microbial Pattern in Acute Exacerbation in Tertiary Care
}

\section{Authors \\ Muthukumar Shunmugam ${ }^{1}$, Joseph Pratheeban ${ }^{2}$, Chandrashekar Madhu ${ }^{3}$ Lekshmi Reghunath ${ }^{4}$}

${ }^{1}$ Assistant Professor, Dept of Thoracic Medicine, Kanyakumari Government Medical College, Kanyakumari

${ }^{2}$ Assistant Professor, Dept of Thoracic Medicine, Kanyakumari Government Medical College, Kanyakumari

${ }^{3}$ Junior Resident, Dept of General Medicine, Kanyakumari Government Medical College, Kanyakumari

${ }^{4}$ Intern, Department of General Medicine, Kanyakumari Government Medical College Hospital

\section{ABSTRACT}

Chronic Obstructive Pulmonary Disease (COPD) is a leading cause of morbidity and mortality worldwide.

The natural history of COPD is characterized by frequent exacerbations. Majority of exacerbations are infectious and knowledge on the pattern of infectious bacteria and their antibiotic sensitivity is essential to formulate cost-effective antibiotic strategy and reducing the emergence of drug resistance. Fifty sputum samples from clinically diagnosed COPD cases suffering from acute exacerbation were collected to isolate and identify the aerobic bacterial agents causing acute exacerbations in COPD patients, to study the antibiotic sensitivity patterns of the isolates and phenotypic detection of ESBL production by the isolates. Preparation of media, reagents, Gram staining, identification of culture isolates, different tests including antibiotic sensitivity tests were carried out following standard laboratory procedures. Klebsiella pneumonia and Escherichia coli were the commonest bacteria isolated followed by Pseudomonas, Citrobacter and anaerobic species ${ }^{(5)}$. All the isolates of K.pneumoniae were ESBL producers. Periodic isolation and identification and resistant status of pathogens causing AECOPD will help us to formulate appropriate treatment protocol and this will be of immense use in reducing mortality and morbidity besides reducing the volume of antibiotics and development of resistance to antibiotics. ${ }^{(6)}$

KEYWORDS: Chronic Obstructive Pulmonary Disease; Antibiotic sensitivity; Pathogenic microbes; ESBL producers.

\section{INTRODUCTION}

Chronic Obstructive Pulmonary Disease (COPD) is a leading cause of morbidity and mortality worldwide. The World Health Organization (WHO) estimated that COPD is currently the seventh leading cause of death and disability worldwide and will become the third leading cause of death worldwide by2020, next to heart and cerebro-vascular diseases. WHO estimates
600 million people worldwide have COPD (World Health Report, 1998). The prevalence of smoking is slowly decreasing in the industrialized world and rising in the developing countries especially in Asia and Africa. This leads to an increase in incidence and prevalence of COPD, although coronary heart disease and stroke show consistent and substantial reduction. This increase is largely driven by two factors namely the rise in tobacco 
related mortality and morbidity and the ageing population ${ }^{(1)}$. Higher prevalence rates for COPD are found in men than in women globally ${ }^{(7)}$. The natural history of COPD is characterized by frequent exacerbations with an increase of cough, purulent sputum production and dyspnoea. Exacerbations punctuate the course of COPD in many patients. Exacerbations of COPD increase the rates of hospitalization and mortality and decrease the quality of life. Majority of exacerbations are infectious in aetiology. Three classes of pathogens responsible for acute exacerbation of COPD by infecting the lower respiratory tract: respiratory viruses, atypical bacteria, and aerobic Gram-positive and Gramnegative bacteria ${ }^{(3)}$. The present study of the aerobic bacterial causes associated with acute exacerbations of Chronic Obstructive Pulmonary Disease (AE COPD) and their in vitro antibiotic sensitivity pattern with special reference to Extended Spectrum Beta - Lactamases (ESBL) production is very pertinent for the clinician to plan a general outline of treatment for the patient with an episode of acute exacerbation of COPD (8). Knowledge on the pattern of local micro organisms and their antibiotic sensitivity is then essential to allow for effective and cost saving antibiotic strategy and reducing the emergence of drug resistance. Therefore a study was undertaken to isolate and identify the aerobic bacterial etiological agents causing acute exacerbations in COPD patients, to study the antibiotic sensitivity patterns of the isolates and phenotypic detection of ESBL production by the isolates ${ }^{(2)}$.

\section{MATERIALS AND METHODS}

\section{Sources of the Material}

Fifty (50) sputum samples from clinically diagnosed COPD cases suffering from acute exacerbation were collected for the study. Patients suffering from bronchial asthma, bronchiectasis, bronchial carcinoma, pneumonia, the subjects who were recently undergoing antibiotic therapy and known cases of pulmonary tuberculosis were not included in the present study.

\section{Data Collection}

Complete data about the patient Name, Age, Sex, Date of collection of the sputum, History of present illness, symptoms were collected from the patient. Past history of smoking, occupational history was also collected. Any history of exposure to indoor air pollution was collected from the female patients.

\section{Sample Collection}

Patient was educated about the difference between sputum and oral secretion. Deeply expectorated sputum sample after an oral gargle with water (to produce a sample from the lower respiratory tract and not getting contaminated with the secretions of the upper respiratory tract) was collected directly into a sterile and wide mouthed disposable universal container. Sample containers were labelled immediately after the collection with patients name, age, sex, IP No, and ICU ward along with date and transported to the Microbiology laboratory. Using these system, negative numbers are assigned to a smear when squamous epithelial cells are observed indicating contamination with oropharyngeal secretions (saliva).

\section{Direct Smear Study}

Direct smears where made from the most purulent part of the sputum samples on a clean grease free microscopic slide and stained with GRAM's STAIN. Under oil immersion objective, the stained sputum smear was observed for the presence of the bacteria, their gram reaction, size, shape, arrangement, any yeast cells, polymorph nuclear leukocytes and squamous epithelial cells. An ideal sputum sample must contain 8-10 polymorphonuclear leucocytes for every 2-3 squamous epithelial cells per high power field. Sputum samples fulfilling these criteria were processed further.

\section{Culture and identification of the isolate}

Sputum samples were plated onto Nutrient agar, Blood agar with Staphylococcus aureus streaks, 
MacConkey's agar, Chocolate agar, Filde's agar. Preparation of media, reagents, Gram staining, identification of culture isolates and different tests were carried out following standard laboratory procedures. The plates are incubated overnight at $37^{0} \mathrm{C}$.After 24 hours of incubation colony morphology was studied. A single well separated colony is identified. Preliminary tests like Gram's staining of the colony, Hanging- drop preparation, Catalase test and Cytochrome oxidase test were done. Biochemical tests like Indole test, Methyl red test, Voges proskauer test, Citrate utilisation test, Urease test, Triple sugar iron agar, Nitrate reduction test, Hugh-Leifson $\mathrm{s}$ oxidation fermentation test, coagulase production (for Staphylococci), Optochin Sensitivity (for Streptococcus pneumoniae) were performed. Sugar fermentation tests with sugars viz: Glucose, Lactose, Sucrose, Maltose, Mannitol, Xylose, Arabinose and Dulcitol, inositols were done to identify the isolate according to standard laboratory procedures.

\section{Analysis of Sample}

Sputum samples from fifty (50) clinically diagnosed cases of AECOPD were analyzed using standard techniques.

Table 1: Bartlett's grading of sputum.

\begin{tabular}{|l|c|}
\hline No. of Neutrophils per 10x & Grade \\
Low Power Field & \\
\hline$<10$ & 0 \\
$10-25$ & 1 \\
$>25$ & 2 \\
Presence of mucus & 1 \\
\hline No. of epithelial cells per 10x & \\
Low power field & -1 \\
\hline $10-25$ & -2 \\
$>25$ & \\
$*$ TOTAL & \\
\hline
\end{tabular}

*Average the number of epithelial cells and neutrophils in about 20 or 30 separate 10x microscopic fields and then calculate the total. A final score of 0 or less indicates lack of active inflammation or contamination with saliva.

\section{Result and Discussion}

The age group of the patient in the study, ranged from forty five to eighty four years. Out of fifty (50) patients, the most common age group was 5574 years $(68 \%)$. The next common age group was 45-54 years(16\%) and 75-84 years (16\%). Among clinically diagnosed COPD patients who were suffering from acute exacerbations, 45 (90\%) were males and $5(10 \%)$ were females. Out of 45 males, all were smokers. Chronic history of cough with expectoration and exertional dyspnoea were the common clinical manifestations in the patients. Majority of the patients had mucopurulent sputum and had a history of aggravated cough with expectoration when exposed to cold climate.

\section{Bacteriological Profile}

Twenty six sputum samples (52\%) were positive for pathogenic bacteria and twenty four samples $(48 \%)$ were culture sterile. Out of the 26(52\%) sputum samples which grew pathogenic bacteria, all samples yielded mono microbial growth. Out of 26 mono microbial growths, Klebsiella pneumonia (30.8\%) and E. Coli (30.8\%) were the commonest bacteria, isolated in 8 cases each, followed by Pseudomonas aeruginosa (26.9\%) in 7 cases, Citrobacter (7.7\%) in 2 cases and anaerobic species $(3.8 \%)$ in 1 case.

COPD is a heterogeneous disease affecting the older and often socially disadvantaged population. It is likely that different insults or susceptibility in combination with smoke exposure are responsible for this heterogeneity. They are a major cause of hospital admission and health care utilization. Large numbers of COPD patients have never been diagnosed or indeed have been misdiagnosed. Further, there has been a dramatic rise in antibiotic resistance among common respiratory pathogens in recent years. Current evidence indicates that chronic infection probably contributes to the pathogenesis of COPD in a substantial subset of patients. Low socioeconomic conditions, poor diet, environmental pollution and childhood infections are not only 


\section{JMSCR Vol||04||Issue||12||Page 15070-15074||December}

responsible for development of COPD but also for continued decline in lung functions, disease complications and an early mortality.

It was observed that AECOPD was prevalent in 45-84 year age group. However among them, 5574 year age group constituted $68 \%$. The probable reason may be because of expression of deterioration in host defences at the bronchial mucosal level in patients with advanced lung disease. AECOPD was higher in males 45 (90\%) than females $5(10 \%)$. The male preponderance was seen in our study because most of them were smokers. In non smokers, especially among women, exposure to indoor air pollution was an important factor. Studies conducted in very different environments have constantly observed that admissions to COPD increased on days with high pollution levels. In the present study AECOPD was more common among smokers than non-smokers which was similar to other studies. It is well known that the frequency of infection resulting in AECOPD by various microorganisms varies from one geographical area to another. Our country has a wide climatic variation and COPD is more common in northern India because of long cold winters, small houses and high levels of indoor pollution. ${ }^{(4)}$

Table 2: Growth pattern in 50 sputum samples

\begin{tabular}{|l|c|c|}
\hline Growth & No. of samples & Percentage \\
\hline Single isolate & 26 & $52 \%$ \\
\hline Multiple isolates & 0 & 0 \\
\hline Non pathogenic & 24 & $48 \%$ \\
\hline
\end{tabular}

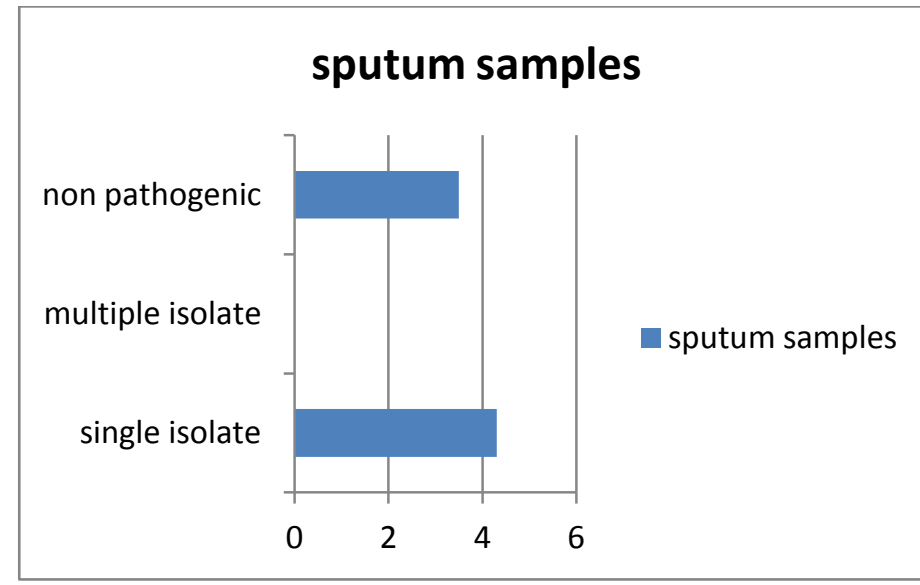

Table 3: Organisms isolated from sputum culture (26 culture positives)

\begin{tabular}{|l|c|c|c|}
\hline S.No & Name of organism & $\begin{array}{c}\text { No. of isolates } \\
(\mathrm{n}-50)\end{array}$ & Percentage \\
\hline 1 & $\begin{array}{c}\text { Klebsiella } \\
\text { pneumoniae }\end{array}$ & 8 & $30.8 \%$ \\
\hline 2 & $\begin{array}{c}\text { Escherichia coli } \\
\text { Pseudomonas } \\
\text { aeruginosa }\end{array}$ & 8 & $30.8 \%$ \\
\hline 3 & Citrobacter & 2 & $26.9 \%$ \\
\hline 4 & anaerobic species & 1 & $3.7 \%$ \\
\hline 5 & \multicolumn{3}{|c}{} \\
\hline
\end{tabular}

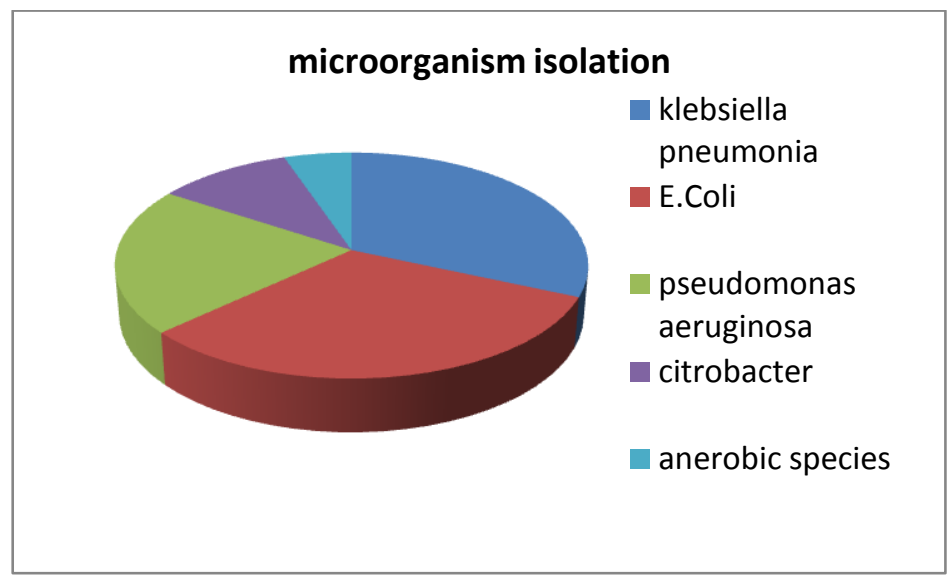

Pathogenic bacteria were found in 52\% of patients with AECOPD. This could be due to declining lung function. Among the isolates, Klebsiella pneumoniae $(30.8 \%)$ and Escherichia coli (30.8\%) were the predominant organisms isolated followed by Pseudomonas aeruginosa (26.9\%), Citrobacter (7.7\%) and anaerobic species (3.8\%). Staphylococcus aureus and $H$. influenzae were not isolated in the present study. It could have occurred because of prior antibiotic use or seasonal variations in causation.

\section{CONCLUSION}

In the present study Klebsiella pneumoniae and $E$. coli showed a higher percentage than other pathogenic bacteria. It is because both the organisms are nosocomial pathogens and large number of patients in our study was the regular patients to the Hospital. Hence the higher percentage of both the organisms could be because of increased oropharyngeal carriage by these patients. It has been shown that the organisms persist in the sputum after apparently effective therapy of acute exacerbations of chronic 
bronchitis and the same phenotypic isolates were responsible for subsequent relapses.

Bacteria play either a primary role in the development of exacerbations of COPD or represent a secondary infection following an initial viral process. The role of bacteria in exacerbations of COPD remains controversial, since bacterial species are present in the airways of between $25-50 \%$ of patients with COPD even when in a stable condition.

Antibiotics are important in treatment of AECOPD. The most common decision a physician has to make when treating a patient with AECOPD is to prescribe the right antibiotic therapy. Antibiotics are commonly prescribed empirically to patients presenting with AECOPD to treat presumed bacterial infection. The rise in bacterial resistance to antibiotics has focused our attention for the purpose of this study. Multi drug resistant strains of the bacteria infect the lower respiratory tract and cause acute exacerbations of COPD. This may result in increase in morbidity and mortality of the patient thereby increasing the economic burden of a nation.

The choice of antibiotics depends on the local antibiotic policy and the pattern of local pathogens. Based on the sensitivity pattern, the common antibiotic that can be used are Amikacin, Ceftazidime-Clavulanic acid, Ceftriaxone, Ciprofloxacin, Co - trimoxazole and Imipenem. In case of mild to moderate AECOPD the patients are to be treated with a short course of antibiotics for a minimum of 5 days than traditional longer treatment.

This study concluded that Klebsiella pneumonia and E. coli were the predominant organisms isolated from the sputum of patients suffering from AECOPD followed by Pseudomonas, Citrobacter and anaerobic species. Periodic isolation and identification and resistant status of pathogens responsible for AECOPD will help us to formulate appropriate treatment protocol and this will be of immense use in reducing mortality and morbidity besides reducing the volume of antibiotics and development of resistance to antibiotics. The protective host immune responses that develop after exacerbation needs to be characterized to facilitate vaccine development

\section{REFERENCES}

1. Allegra, L., N. Konietzko, P, Leophonte, et al. 1996. Comparative safety and efficacy of sparfloxacin in the treatment of acute exacerbations of chronic obstructive pulmonary disease: a double-blind, randomised, parallel,multicentre study. J Antimicrob Chemother. 37(Suppl A):93 104.

2. American Lung Association. 2011.Chronic Obstructive Pulmonary Disease (COPD) Fact Sheet. February 2011. Http://www.lung.org /lungdisease/copd/resources/factsfigures/COPD-Fact-Sheet.html.

3. Arora, N., M.K. Daga et al. 2001. Microbial pattern of Acute Infective Exacerbation of Chronic Obstructive Airway Disease in a Hospital Based Study .Indian Chest Dis . Allied Sci.43:157-162.

4. Chan Yeung, M., N.Ait Khaled, N.White, M.S. Ip and W.C Tan. 2004. The Burden and impact of COPD in Asia and Africa. Int .J. Tuber Lung. Dis. 8(1):2-14

5. Chronic Obstructive Pulmonary Disease (COPD). Available at: Http://www.patient.co.uk/health/chronic-obstructivepulmonary-disease

6. Erkan, L., Uzun O, Findik S et al., 2008. Role of bacteria in acute exacerbations of chronic obstructive pulmonary disease, Int J Chron Obstruct Pulmon Dis. 3: 463467.

7. Global Strategy for the diagnosis, management and prevention of COPD.2011. (Revised2011); 1:1-7.

8. Iyer Parameswaran, G., and Murphy, T.F. Chronic obstructive pulmonary disease: role of bacteria and updated guide to antibacterial selection in the older patient. 